\title{
Large Cell Neuroendocrine Tumor of the Lung: A Diagnostic Challenge
}

\author{
Jayasri Helen Gali, Harsha Vardhana Varma \\ Department of Pulmonology, Apollo Institute of Medical Sciences and Research, Hyderabad, Telangana, India
}

\section{Abstract}

Lung neuroendocrine carcinoma (NEC) is a rare heterogeneous carcinoma with NE cells in the pulmonary and bronchial epithelium as the site of origin. We describe a case of large cell NEC (LCNEC) in a 55-year-old male, smoker with 25 pack-years, who presented with cough, dyspnea, hemoptysis, and loss of appetite for 6 months. There was no diabetes, hypertension, asthma, chronic obstructive pulmonary disease, tuberculosis, or contact with tuberculosis or malignancy. The presence of a large homogenous mass lesion in the right chest on chest X-ray, further clearly defined on chest computed tomography scan $(130 / 94 / 123 \mathrm{~mm})$ was suggestive of huge mass. There was no mediastinal or pleural involvement. Possibility of LCNEC was suspected as the ultrasound-guided biopsy was indicating the presence of poorly differentiated carcinoma. Immunohistochemistry was negative for thyroid transcription factor, cytokeratin 7 but was positive for synaptophysin and P40, suggestive of high-grade LCNECs. It was in T4N0M0, stage 3A and treatment with combination chemotherapy was initiated.

Keywords: Immunohistochemistry, large cell neuroendocrine tumor, lung neuroendocrine tumor

\section{INTRODUCTION}

Lung neuroendocrine carcinoma (NEC) is a rare heterogeneous carcinoma with NE cells in the pulmonary and bronchial epithelium as the site of origin, accounting for $20 \%$ of all lung cancers. It is estimated that $\approx 3 \%$ of NECs are large cell NECs (LCNECs). ${ }^{[1]}$ Awareness regarding lung NECs is low among medical fraternity. We present a case of LCNEC of the lung that was initially unsuspected and undiagnosed.

\section{Case Report}

A 55-year-old gentleman, driver, presented with cough, dyspnea, hemoptysis, and loss of appetite for 6 months. Cough was productive with white mucoid scanty sputum and occasional streaky hemoptysis. Dyspnea was gradual in onset, progressive, Grade 2 on modified Medical Research Council dyspnea scale, not associated with wheeze and aggravated on lying on the left lateral position. He had loss of appetite (2 months) and weight loss (3-4 kg in the last 1 month); there was no chest pain. There was no past/present history of contact with tuberculosis. He was a smoker with 25 pack-years and consumed alcohol occasionally. He had no

\begin{tabular}{|l|l|}
\hline \multicolumn{2}{|c|}{ Access this article online } \\
\hline Quick Response Code: & Website: \\
& www.ijrconline.org \\
\hline
\end{tabular}

history of chronic systemic diseases (diabetes, hypertension) or other respiratory illness (asthma, chronic obstructive pulmonary disease, and malignancy). There was no family history of tuberculosis or malignancy. He was evaluated as an inpatient for about 20 days in a private hospital; a large homogenous mass in the right chest [Figure 1] was noted on chest X-ray (CXR), which was further clearly defined on chest computed tomography scan (CT scan). It measured about 130/94/123 mm without any mediastinal or pleural involvement [Figures 2 and 3]. Fiberoptic bronchoscopy was normal, and the bronchoalveolar lavage was negative for malignant cells; Gram stain, and culture and sensitivity, smear for acid-fast bacilli and Cartridge-Based Nucleic Acid Amplification Test were negative. As there was no evidence of tuberculosis or malignancy, he received symptomatic treatment. He consulted us for the second opinion as the symptoms persisted.

Address for correspondence: Dr. Harsha Vardhana Varma, Department of Pulmonology, Apollo Institute of Medical Sciences and Research, Jubilee Hills, Hyderabad, Telangana, India. E-mail: harsha.varmap@gmail.com

This is an open access journal, and articles are distributed under the terms of the Creative Commons Attribution-NonCommercial-ShareAlike 4.0 License, which allows others to remix, tweak, and build upon the work non-commercially, as long as appropriate credit is given and the new creations are licensed under the identical terms.

For reprints contact: reprints@medknow.com

How to cite this article: Gali JH, Varma HV. Large cell neuroendocrine tumor of the lung: A diagnostic challenge. Indian J Respir Care 2020;9:97-9.

Received: $28-02-2019$

Accepted: $20-05-2019$
Revised: $14-05-2019$

Published: 08-01-2020 
Gali and Varma: Large cell neuroendocrine tumor

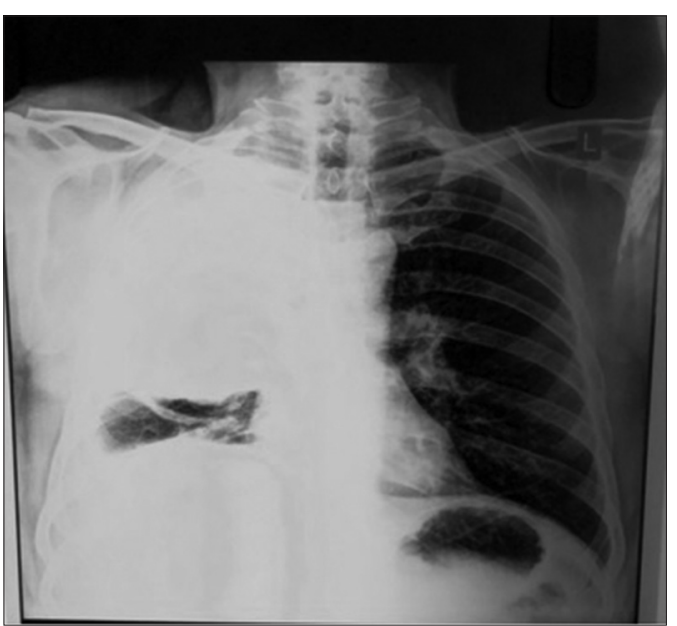

Figure 1: Chest X-ray - posterior-anterior view - large homogenous mass lesion in the right chest

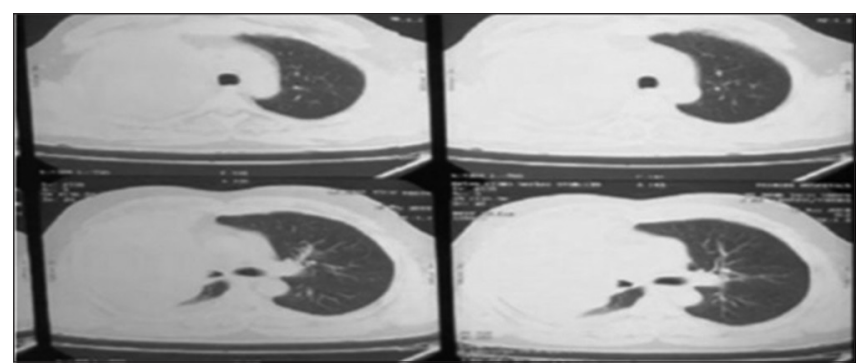

Figure 3: Computed tomography scan - chest-lung window - large homogenous mass lesion right chest, no pleural effusion

He was moderately built and nourished, without any clubbing or lymphadenopathy on general examination. Vitals were stable, the trachea was centrally placed, reduced chest movements of the right side and total chest expansion, decreased tactile vocal fremitus, dull percussion note on the right chest with reduced breath sounds were noted. As the CXR and CT scan chest showed a large mass touching the right chest wall, ultrasound-guided biopsy of the lung mass was done, and the histopathology revealed features of poorly differentiated carcinoma with possibility of large cell carcinoma [Figure 4].

Immunohistochemistry (IHC) was negative for thyroid transcription factor (TTF), cytokeratin 7 and positive for synaptophysin [Figure 5] and P40, suggesting high-grade LCNEC. He was referred to the oncology department for chemotherapy. As it was a large tumor and was of T4N0M0, stage 3A, he was started on combination chemotherapy (platinum and etoposide regimen). He responded to the treatment initially, with clinical improvement, but was lost to follow-up after 3 months.

\section{Discussion}

In the 1970s, lung cancers were classified based on histological features. ${ }^{[2]}$ Travis et al..$^{[3]}$ defined LCNEC with characteristic, diagnostic histological features. Till date, very few reports of lung LCNEC are available from India. ${ }^{[4,5]}$

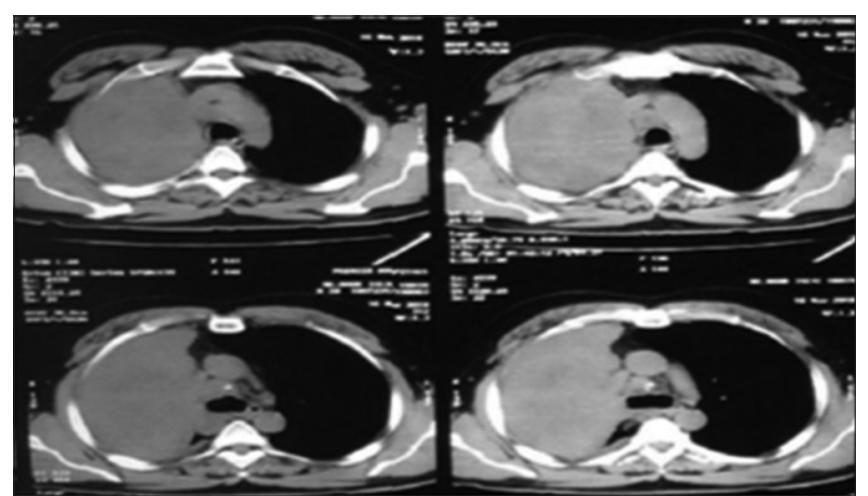

Figure 2: Computed tomography scan chest - mediastinal cut - large homogenous mass lesion 130/94/123 mm, no mediastinal invasion

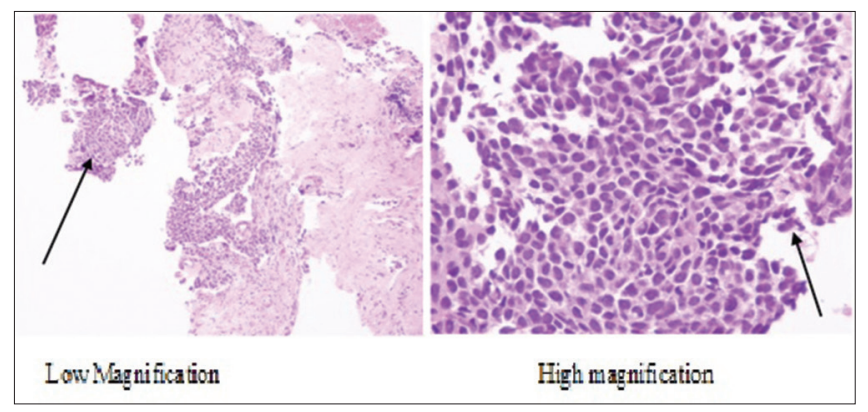

Figure 4: Large neoplastic cells with hyperchromatic nuclei and scant cytoplasm ( $\mathrm{H}$ and $\mathrm{E})$

The LCNEC tumors of lung occur commonly in the elderly (median age, 65 years), males, and heavy smokers. They are localized peripherally with a high recurrence rate, poorly symptomatic at diagnosis. Cough, hemoptysis, or postobstructive pneumonia is uncommon. They commonly present with an asymptomatic nodule or chest pain, nonspecific flu-like symptoms, dyspnea, night sweats, and rarely as carcinoid or paraneoplastic syndromes. Being a highly metastatic tumor, survival of the patient depends on the rate of its spread; Involvement of the lymph node which is seen in $60 \%-80 \%$ of patients and distant metastasis ( $40 \%$ ) are associated with poor survival rates.

Lung LCNEC shares several clinicopathological features with small cell lung cancer (SCLC) and postulated to represent its variant form. It is also essential to distinguish LCNEC from poorly differentiated non-SCLC (NSCLC) and typical and atypical carcinoids. LCNEC has a poor prognosis compared to other NSCLC at stage 1 disease, which is almost comparable with that of patients with SCLC.

LCNEC generally presents as an expanding mass having irregular margins, which is peripherally located, without any specific finding on CXR or CT. The characteristic histological features were not demonstrated in our case. As LCNEC closely mimics SCLC, atypical carcinoid, or NSCLC in biopsy/cytology specimen, differentiating these is a difficult task; while the small samples only suggest a diagnosis of lung LCNECs, IHC is often required for confirmation. Established NE markers include chromogranin A, synaptophysin, and CD56. 
Gali and Varma: Large cell neuroendocrine tumor

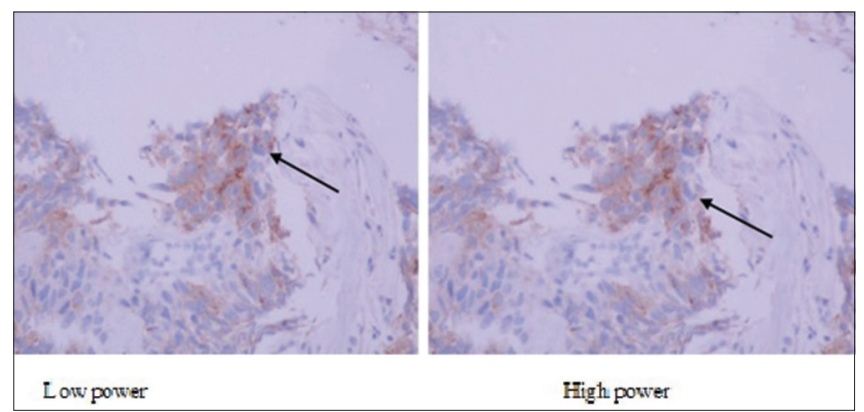

Figure 5: Neoplastic cells with focal synaptophysin positive (IHC, low and high power)

In 1991 Travis et al $\cdot{ }^{[3]}$ reported that the prognosis of LCNEC is between that of atypical carcinoids and SCLC. LCNEC was described as having a cell size at least three times that of SCLC, an organoid growth pattern, cellular-palisading or rosette-like areas, a high mitotic rate, and variable granular chromatic pattern. ${ }^{[6]}$

Typical NE markers, such as chromogranin, neuron-specific enolase, synaptophysin, and somatostatin, are secreted by LCNECs. Biomarkers, i.e., high-molecular-weight cytokeratins, typically expressed by SCLC and other NE tumors, ${ }^{[1]}$ make the diagnosis easier while the conspicuous absence of characteristic markers for LCNECs often poses a difficulty in diagnosis of the latter. ${ }^{[7]}$ Our patient was positive for synaptophysin, TTF, and chromogranin and negative for cytokeratin 7 , cytokeratin, and napsin. Takei et al. ${ }^{[8]}$ reported positive IHC staining with chromogranins (82\%), using synaptophysin (91\%), and neural cell adhesion molecule with no marker expressed in LCNEC cases (91\%) and LCNECS expressing all three markers (68\%). Positive NE marker (synaptophysin, chromogranin A, or CD56) is sufficient to confirm the diagnosis, but CD56 expression alone requires cautious interpretation. ${ }^{[9]}$

The National Cancer Control Network recommends treatment for LCNEC according to the NSCLC guidelines; often, the drugs used are the same for both LCNEC and SCLC; reports also suggest that LCNEC is less chemoresponsive to platinum/etoposide regimen unlike SCLC with approximately $50 \%-80 \%$ response rates. ${ }^{[10]}$

Although highly precise techniques resulted in diagnostic accuracy, delayed/missed diagnosis still remains a challenge in lung LCNEC as many are diagnosed in autopsy specimen. All stage 5-year survival rates for LCNEC range from $13 \%$ to $57 \%,{ }^{[9]}$ making it worthwhile to invest in increasing the public awareness of LCNEC to improve the treatment-seeking behavior.

\section{Conclusion}

Lung LCNEC requires clinical and pathological expertise for diagnosis and must be considered as a possibility in all poorly differentiated lung cancers. Rarity, aggressive nature, and extremely poor prognosis, comparable with SCLC, demand thorough clinicopathological evaluation and combined modalities of treatment.

\section{Declaration of patient consent}

The authors certify that they have obtained all appropriate patient consent forms. In the form the patient(s) has/have given his/her/their consent for his/her/their images and other clinical information to be reported in the journal. The patients understand that their names and initials will not be published and due efforts will be made to conceal their identity, but anonymity cannot be guaranteed.

\section{Acknowledgement}

We thank Dr. M. S. Latha for editing and proofreading.

\section{Financial support and sponsorship}

Nil.

\section{Conflicts of interest}

There are no conflicts of interest.

\section{RefEREnCes}

1. Fasano M, Della Corte CM, Papaccio F, Ciardiello F, Morgillo F. Pulmonary large-cell neuroendocrine carcinoma: From epidemiology to therapy. J Thorac Oncol 2015;10:1133-41.

2. Arrigoni MG, Woolner LB, Bernatz PE. Atypical carcinoid tumors of the lung. J Thorac Cardiovasc Surg 1972;64:413-21.

3. Travis WD, Linnoila RI, Tsokos MG, Hitchcock CL, Cutler GB Jr., Nieman L, et al. Neuroendocrine tumors of the lung with proposed criteria for large-cell neuroendocrine carcinoma. An ultrastructural, immunohistochemical, and flow cytometric study of 35 cases. Am J Surg Pathol 1991;15:529-53.

4. Amirtham U, Mulchandani NJ, Premalata CS, Bharatnur SA, Kumar RV. Spectrum of primary pulmonary neuroendocrine tumors from a regional cancer centre in South India. J Med Sci Clin Res 2017;5:24997-5002.

5. Mohapatra P, Patra S, Bhuniya S, Panigrahi M, Mishra P, Purkait S, et al. Pulmonary large cell neuroendocrine carcinoma (LCNEC): An experience from Eastern Indian hospital. J Thorac Oncol 2017;12:S2183-4.

6. Battafarano RJ, Fernandez FG, Ritter J, Meyers BF, Guthrie TJ, Cooper JD, et al. Large cell neuroendocrine carcinoma: An aggressive form of non-small cell lung cancer. J Thorac Cardiovasc Surg 2005;130:166-72.

7. Iyoda A, Hiroshima K, Nakatani Y, Fujisawa T. Pulmonary large cell neuroendocrine carcinoma: Its place in the spectrum of pulmonary carcinoma. Ann Thorac Surg 2007;84:702-7.

8. Takei H, Asamura H, Maeshima A, Suzuki K, Kondo H, Niki T, et al. Large cell neuroendocrine carcinoma of the lung: A clinicopathologic study of eighty-seven cases. J Thorac Cardiovasc Surg 2002;124:285-92.

9. Hiroshima K, Mino-Kenudson M. Update on large cell neuroendocrine carcinoma. Transl Lung Cancer Res 2017;6:530-9.

10. Naidoo J, Santos-Zabala ML, Iyriboz T, Woo KM, Sima CS, Fiore JJ, et al. Large cell neuroendocrine carcinoma of the lung: Clinico-pathologic features, treatment, and outcomes. Clin Lung Cancer 2016;17:e121-9. 